\title{
Polysèmes
}

Revue d'études intertextuelles et intermédiales

\section{Pli des temps et refuge des images : travail du montage dans Le Petit Chaperon rouge re-conté par Sarah Moon}

In the Fold of Time: Montage in Little Red Riding Hood Re-told by Sarah Moon

\section{Olivier Leplatre}

\section{(2) OpenEdition}

Journals

Édition électronique

URL : http://journals.openedition.org/polysemes/2296

DOI : $10.4000 /$ polysemes.2296

ISSN : 2496-4212

Éditeur

SAIT

Référence électronique

Olivier Leplatre, «Pli des temps et refuge des images : travail du montage dans Le Petit Chaperon rouge re-conté par Sarah Moon », Polysèmes [En ligne], 18 | 2017, mis en ligne le 30 novembre 2017, consulté le 19 avril 2019. URL : http://journals.openedition.org/polysemes/2296 ; DOI : 10.4000/ polysemes.2296

Ce document a été généré automatiquement le 19 avril 2019.

Polysèmes 


\section{Pli des temps et refuge des images : travail du montage dans Le Petit Chaperon rouge re-conté par Sarah Moon}

In the Fold of Time: Montage in Little Red Riding Hood Re-told by Sarah Moon

\section{Olivier Leplatre}

«J'invente une histoire que je ne raconte pas »

(Sarah Moon 1991)

En 1983, Charles Perrault et Sarah Moon «cosignent» un album paru dans la toute nouvelle collection «Monsieur chat» lancée par Grasset-Jeunesse (fig. 1). L'ouvrage reprend Le Petit Chaperon rouge publié par Perrault en 1698. À cette date, le conte se découvrait accompagné d'une vignette et l'ensemble du recueil s'ouvrait sur un frontispice dont l'écrivain lui-même avait certainement fourni l'original (fig. 2). Mais l'entreprise de Sarah Moon est plus ambitieuse : la photographe choisit d'enrichir le texte d'une dizaine de clichés en noir et blanc. Elle relit ainsi en images le conte dont, comme elle a pu le dire, elle invente l'histoire sans la raconter, un conte en somme qu'elle reconte, tissant à son tour pour les yeux la vieille trame des nourrices. 
Le Petit Chaperon Rouge

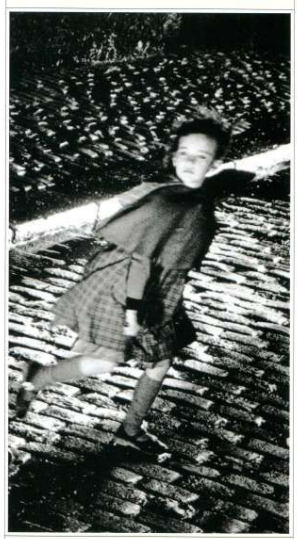

Chaki.es Perrault ILLSTRE PAR SARAH MOON

Fig. 1 : Le Petit Chaperon rouge de Charles Perrault, illustré par Sarah Moon, collection « Monsieur chat », Grasset-Jeunesse, 1983, (C) Sarah Moon

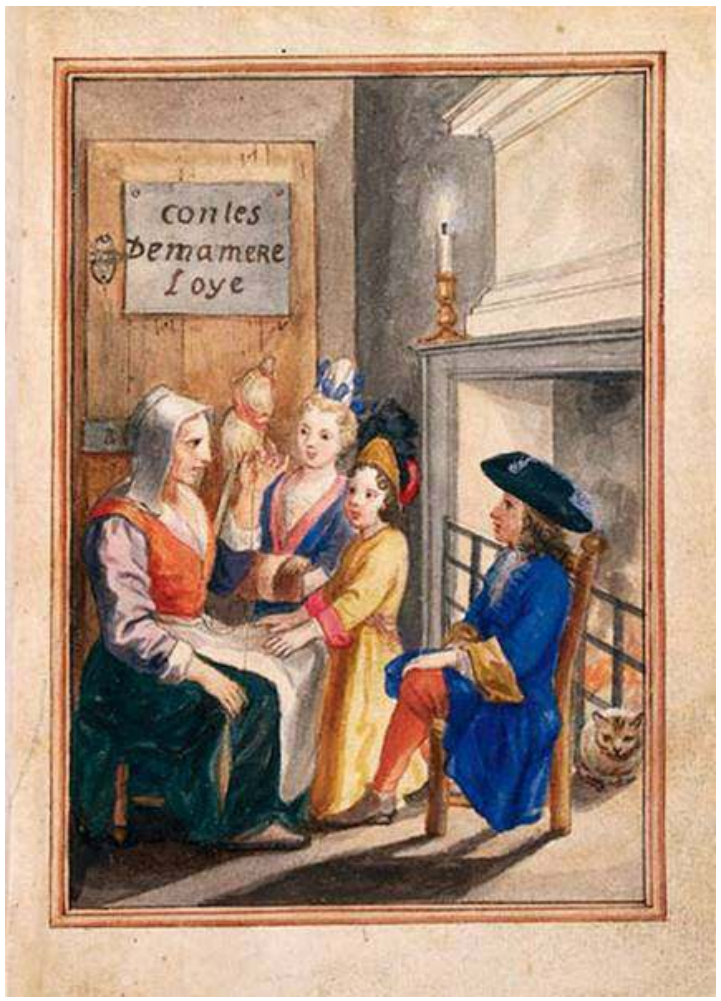

Fig.2 : Frontispice des Contes de ma Mère Loye (1698), Charles Perrault, Wikimedia Commons

2 Le frontispice prévu par Perrault au seuil de sa version des Contes de ma Mère Loye rapprochait en une même unité sensorielle les gestes de la conteuse ; il faisait en effet coïncider plastiquement le filet de voix sortie de la bouche, les doigts pinçant la laine et le 
regard, foyer de l'imagination. Derrière le visage de celle qui, en dévidant le récit, prépare métaphoriquement les textes que nous nous apprêtons à lire, exactement au niveau de son œil grand ouvert, le trou de la serrure promet de stupéfiantes images (fig. 3); elles bruissent de mots dont elles attendent que l'écoute les libère.

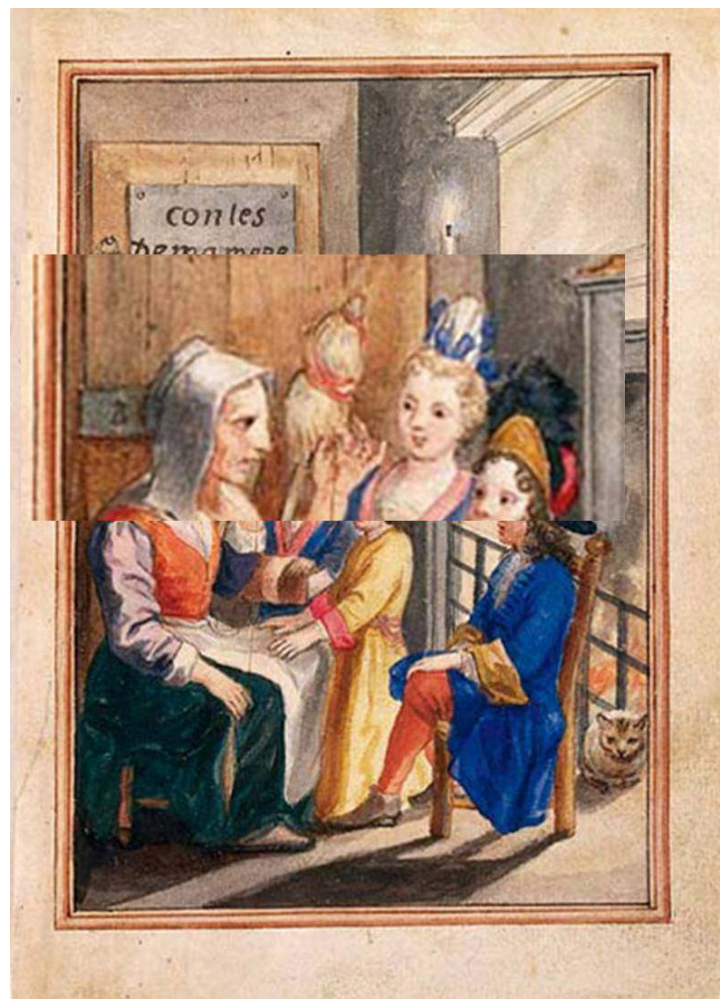

Fig.3 : Le trou de la serrure derrière le visage de la conteuse

3 Au même endroit apparemment, Sarah Moon accroche une horloge (fig. 4). Elle est répétée dans l'album (fig. 5) de façon à marquer la cadence des images et de l'histoire qu'elles escortent. Dans une des premières images, ce cadran, incrusté (fig. 6) ou en relief, est redoublé par le rectangle sombre d'une page du conte dactylographiée qui a été négligemment collée à un support de planches au relief granuleux. Engrenés ensemble, les rouages du cadran et de la page actionnent une étrange petite machine. Elle est destinée à fabriquer les images. C'est un appareil photographique, primitif, bricolé de bric et de broc, de temps et de texte. Aussi la porte de la nourrice, approchée de très près pour pouvoir entrer dans les secrets qu'elle tient invisibles, se mue-t-elle en pan visuel; au déclenchement du retardateur, il est cinq heures (du matin ou du soir), l'œil-temps du cadran remplit l'orbite de la serrure, et débute l'œuvre de remontage voulu par la photographe. 


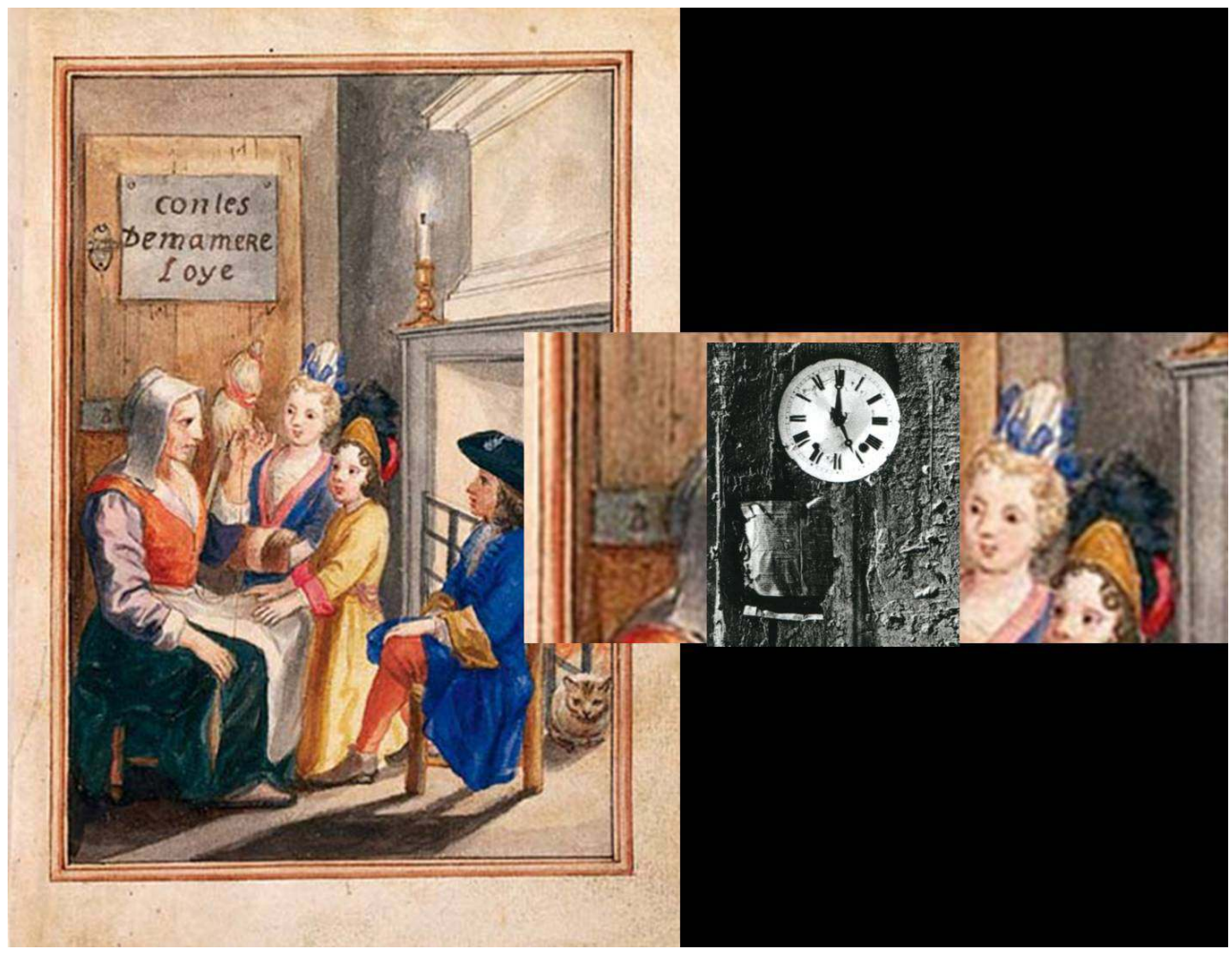

Fig.4 : Au même endroit, Sarah Moon accroche une horloge, (c) Sarah Moon

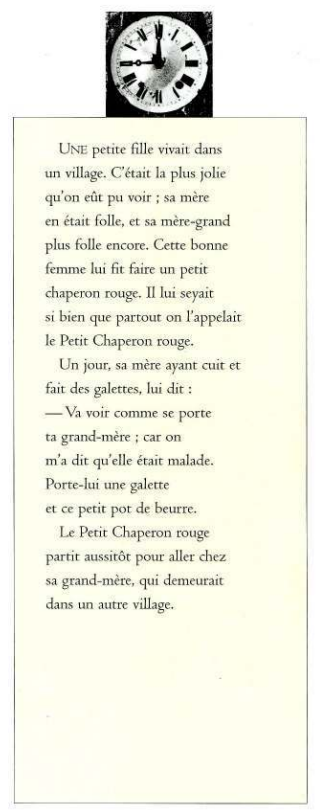

Fig. 5 : L'horloge marque la cadence des images et de l'histoire, (c) Sarah Moon 


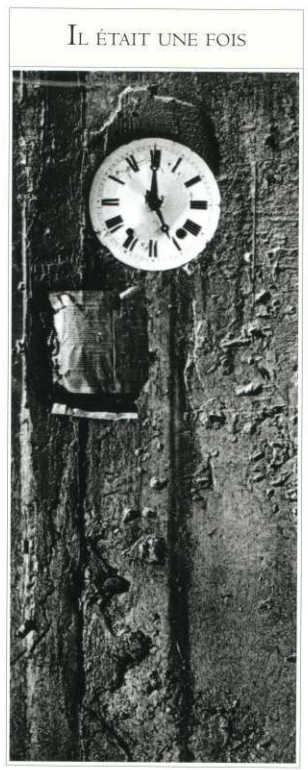

Fig.6 : Le cadran de l'horloge est redoublé par le rectangle sombre d'une page du conte dactylographiée, (c) Sarah Moon

4 Sarah Moon procède à diverses interventions sur le texte de Perrault: elle en modifie ponctuellement la lettre ; surtout, pour fabriquer son album (le mot convient à ce travail qui relève du livre d'artiste et de l'ouvrage pour la jeunesse), elle fragmente en séquences le conte originel. Dans le texte, que sous cet angle elle réécrit, elle aménage des intervalles dans lesquels glisser ses photographies. Combinées à l'écriture pour la couverture et pour la page de titre, les images sont distinctes du récit, mis en page quant à lui au sein d'un cadre surmonté de l'horloge dont les aiguilles avancent (fig. 7). Dans l'économie compositionnelle de l'album, les photographies ont tendance à déborder et à s'émanciper. Une première image pleine page et hors texte introduit le chaperon, jailli et presque accouché de l'ombre farouche des arbres (fig. 8); puis ce sont deux photographies qui sont insérées entre chaque partie du texte; elles sont le plus souvent disposées sur une double page jusqu'à leur acmé dramatique, l'agression supposée de l'enfant (fig. 9). Pour cette dernière photographie, un drap réunit dans un même froissement page et image ; son écran plissé vient barrer le regard et fermer le livre. 


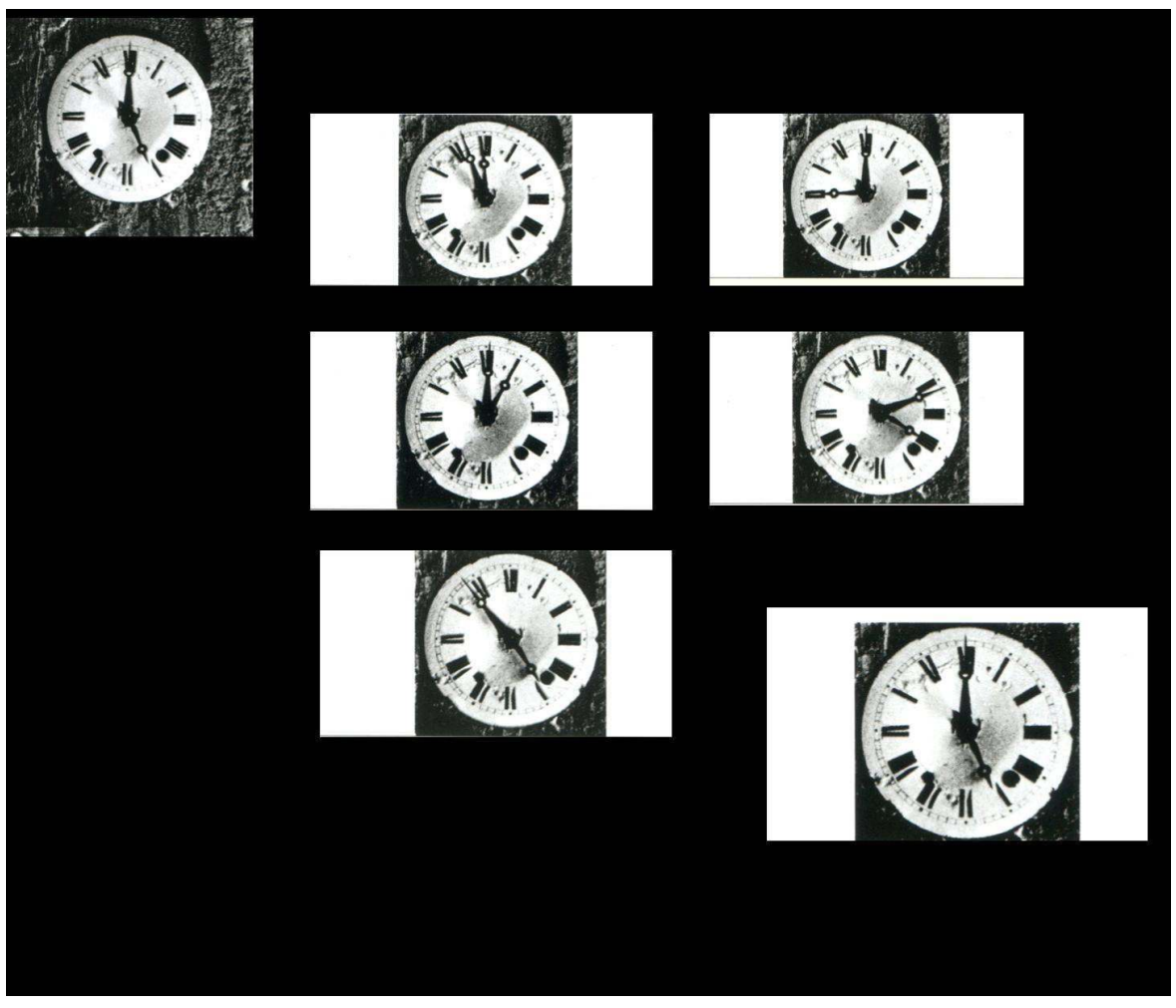

Fig.7 : Les aiguilles avancent, (c Sarah Moon

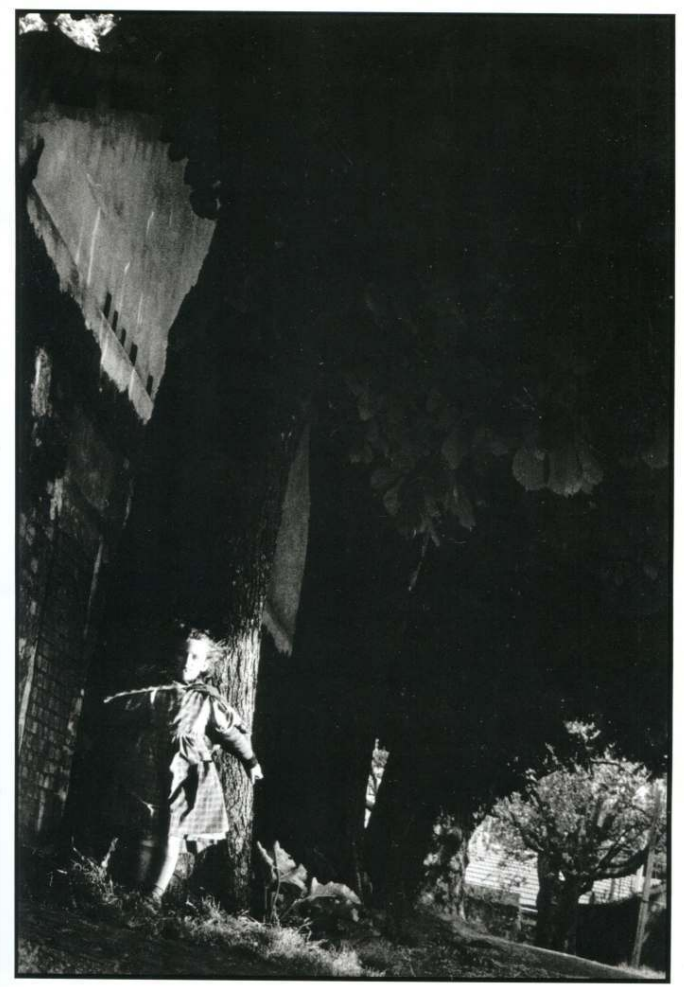

Fig.8 : Première image, pleine page et hors texte, qui introduit le chaperon, (c) Sarah Moon 


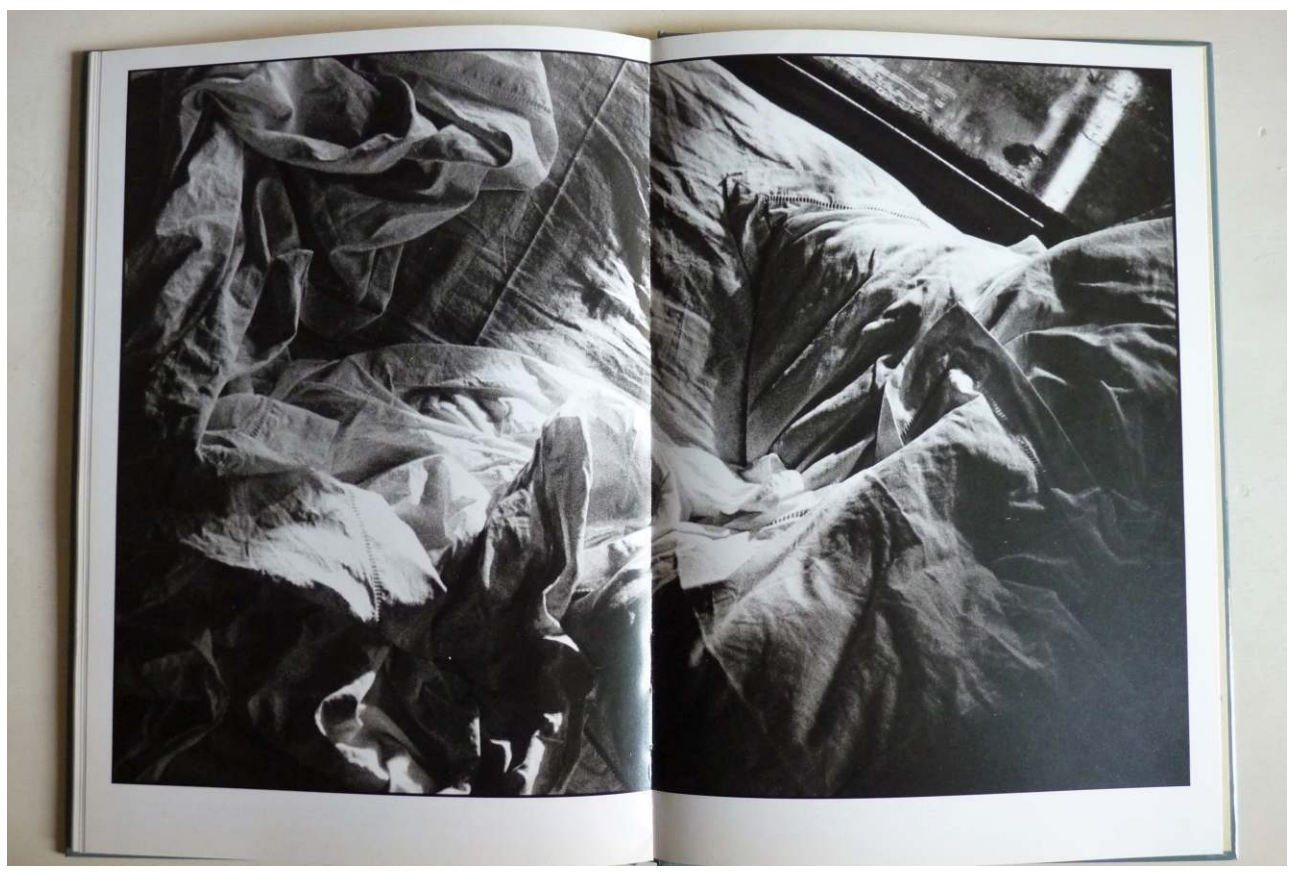

Fig.9 : Dernière photographie : un drap réunit dans un même froissement page et image, @ Sarah Moon

5 Pour exprimer en images le conte de Perrault, la lecture de Sarah Moon retrouve ce que Giorgio Agamben isole comme les conditions de possibilités du cinéma et qui, en l'occurrence, s'applique à la photographie. Quels sont, s'interroge Agamben, les transcendantaux du cinéma? La répétition et l'arrêt (Agamben 2004). La répétition offre le retour en possibilité de ce qui a été et qui, s'agissant ici de photographier Le Petit Chaperon rouge, fait advenir dans le texte de Perrault l'une de ses virtualités, déposée en latence au creux de l'imagination puis éveillée par la lumière de la lecture traduite en exposition photographique. Devenu réalité d'images, le projet de Sarah Moon célèbre ainsi dans le conte la fécondité de son inaccomplissement essentiel et sa capacité, en raison de sa puissance de sollicitation psychique, à produire des bouffées d'images.

6 L'autre transcendantal du cinéma, selon Agamben, est l'arrêt, qui le rattache intrinsèquement à l'intention photographique mais le rapproche aussi de la poésie. Non l'arrêt «au sens d'une pause, chronologique, c'est plutôt une puissance d'arrêt qui travaille l'image elle-même, qui la soustrait pour l'exposer en tant que telle » (Agamben 93). En morcelant le conte et en le contant de nouveau, photo par photo, Sarah Moon provoque cette hésitation entre le sens et l'image, qui ressemble à ce qu'effectue la césure dans le poème. En stoppant le texte, l'image prend position, elle s'installe en lui ; elle complique alors la lisibilité dans le but avoué de redonner au conte sa pleine singularité (en quoi il me concerne, personnellement et encore maintenant) et elle remet en mouvement le sens.

7 Lire pour Sarah Moon aura d'abord consisté à délier le texte, à interrompre son flux narratif, à trancher le fil de la quenouille pour que se détachent et qu'en une autre langue s'épèlent des images; ou, inversement, les images auront surgi en déchirant le texte, en écartant ses pages. Cette poussée du visible introduit dans le récit, greffé sur lui et faisant irruption sur sa scène, relève d'un régime du lire moins intellectuel que pathique. Elle exprime ce qu'a été le bouleversement d'une lecture à l'évidence intime. Par à-coups, l'écriture a provoqué son événement dans le sensible. Quelque chose a «fait tilt ", pour 
emprunter à Barthes cette expression ludique qui laisse entendre à travers le bruit du flipper le déclic du déclenchement photographique et celui du verrou des rêves et des souvenirs ${ }^{1}$. Par ses détails, ses lignes de force, ses facultés de résonance imaginaire et sensible, qui malgré le connu continuent de sonder l'inconnu (tel est l'entêtement du texte à être encore là), le conte appelle sa mise en image. Son irrésistible attraction, son aimantation, réclame son animation scandée par chaque prise de vue qui aura d'abord eu lieu comme une saisie en soi. Puis la série a entraîné les images pour qu'à travers elles, se manifestent l'insistance de la lecture et l'élan de son émotion. Les photographies portent témoignage d'assauts affectifs, de symptômes sensoriels venus de la lecture du texte et convertis ensuite en visions, aux confins du rêve et de la réalité. Il arrive qu'on lise, note Barthes, en levant les yeux, «non par désintérêt, mais au contraire par afflux d'idées, d'excitations, d'associations?» (Barthes 1993-95, 961). Sarah Moon lève les yeux pour se placer face au prisme de l'objectif, en quête des images que sa lecture a éveillées et pour obtenir, en faisant plus ou moins le point, leur forme vibrante.

L'on transposerait volontiers sur cet effet du conte ce que Raymond Bellour confie à propos des images de cinéma :

On ne choisit pas ses moments. Ils vont, viennent en vous au gré de la mémoire criblée de hasard autant que du désir d'inventorier et de prouver, de réactiver un choc plus ou moins ancien pour l'offrir en partage. Il y a tant de tels moments, tant et tant de plans de cinéma. Tant d'émotions qui s'y attachent, presque inconnues jusqu'à l'instant d'un choix qui soudain les attire et va leur insuffler une vie nouvelle et quasi excessive, tenant à leur valeur d'exemple. Ces moments vous choisissent, vous ont choisi un jour avant de devenir peut-être les motifs d'une argumentation. (Bellour 223)

9 Les photographies se regardent et créent ensemble une pellicule de vues, et elles nous regardent comme autant de révélations affectives liées «à une mise en mouvement qui agite, fragilise le monde intérieur aussi bien que le monde extérieur » (Cérès 10, cité par Vally). À l'arraisonnement du sensible par le récit, l'image répond alors en manifestant à son tour l'envie de détourner le cours de l'écriture. Lors de la lecture, le désir a été sollicité, fixé et ces arrêts en soi sous le coup du texte, l'image les reprend à son compte pour en faire quelque chose, autre chose, de l'ordre d'une réincorporation ou d'un prolongement qui exploite et accroît la force rémanente de l'interruption.

Dans cette photographie par exemple (fig. 10), la légende qui cite le texte relate la course du loup et la promenade du chaperon. Mais l'image, elle, ne montre aucun mouvement. $\mathrm{Au}$ contraire, elle cueille l'enfant, elle le freine ; et l'enfant tourne le regard vers l'œil qui la suit du haut de l'arbre et se confond avec le point de vue du photographe relayant le narrateur du conte. Tout se fige, et l'on a l'impression que ce qui importe est moins de transcrire un moment du récit que de manifester la pulsion d'image qu'il a stimulée, la manière que le texte a eue de lever les yeux en direction de l'image. 




Fig. 10 : «Le loup se mit à courir de toute sa force par le chemin qui était le plus court, et la petite fille s'en alla par le chemin le plus long... », @ Sarah Moon

Sarah Moon a parlé de cette sensibilité photographique aiguë dans les termes d'une fascination pour l'enfance, pour la fragilité de son innocence et la " douceur des choses » auxquelles prendre garde. «Je photographie, confie-t-elle, le privilège - l'évanescence improbable de la beauté - j'y cherche l'émotion et la quête en est d'autant plus désespérante" (Moon 1991). Chaque photographie est vécue par elle comme une tentative pour effleurer la beauté fugitive, tragique parce qu'elle est toujours menacée, inquiétée par l'éventualité de sa scandaleuse profanation, mais capable aussi de défier de sa fraîcheur inaltérable la violence ennemie.

En définitive, le conte ne se trouve pas réinterprété, il n'est même pas vraiment illustré. La photographe cherche à être fidèle moins à la réalité référentielle du texte qu'à l'énergie onirique et fantasmatique animée et développée par le récit, et que renforce encore chacune des séquences cadrées par le montage. Sarah Moon enregistre, dans un acte photographique à la fois très contrôlé et lâché, l'écho que la fiction a exercé sur son imaginaire et la singulière vitalité, l'ébranlement profond dont l'onde de choc a donné naissance à l'image. On retrouve dans cette surprise et cet accaparement des images la répercussion d'une appréhension flottante de la lecture, analogue à l'écoute détachée privilégiée par Freud pour mieux entendre ce que la parole de ses patients pouvait receler.

En résulte un film d'images syncopées, plus ou moins étroitement raccordées, qui racontent leur propre histoire le long du conte et sur son bord. Leur rythme sauté leur confère, page après page, l'allure d'un court-métrage des premiers temps du cinéma ponctué par des cartons où le texte de Perrault prend sa place. Sarah Moon a toujours déclaré son goût pour le cinéma expressionniste et l'album qu'elle a conçu hérite de son esthétique. Mais le film du chaperon rouge reste en-deçà de l'image-mouvement (plus tard, Sarah Moon reviendra au même conte pour oser à partir de lui un véritable film intitulé cette fois Le Petit Chaperon noir ${ }^{2}$ ). Mais, au départ, l'image photographique flirte avec le cinéma sans s'y abandonner, comme s'il s'agissait, en maintenant le principe du 
montage suspendu, de préserver une sorte de sidération continue. Cette sidération se divise entre l'effroi de la violence (l'arrêt suggère un film manquant dont ne seraient plus perceptibles que des fragments, lambeaux ou ruines) et le magnétisme exercé par la beauté qui passe, animée par le visage lumineux du chaperon où Sarah Moon continue de voir dans les interviews qu'elle a données l'étincelle des yeux bleus de Morgan, son jeune modèle à qui l'album est dédié. Au lieu que la photographie ne soit que chute (image tombée sous le coup de la violence, image mordue par les dents du loup), sa puissance de surgissement donne accès à des épiphanies. À chaque déclenchement de l'appareil, elle rend possible une apparition compensant la mélancolie du " cela a été » par le bonheur de ressentir le «c'est ça !» d'où l'image procède et qu'elle valide : «Tel serait le "destin" de la Photographie : en me donnant à croire (il est vrai, une fois sur combien?) que j'ai trouvé "la vraie photographie totale", elle accomplit la confusion inouïe de la réalité ( “Cela a été") et de la vérité (“C'est ça!") » (Barthes 1980, 175-176).

À l'intérieur du film virtuel, les photographies ou les «photogrammes » procèdent, eux, d'un contact plus exact, quoique flottant, avec les stases du récit. À plusieurs reprises, un extrait du texte souligne l'endroit où, dans le récit, l'image semble avoir jailli, le lieu exact de son punctum émouvant et aigu. Ce point de rencontre reste toujours lâche. Les italiques, privilégiées pour la typographie de la légende, indiquent de l'histoire qu'elle est de passage pour insuffler un dynamisme; le rapprochement du texte et de l'image se veut avant tout ouvert à la fluidité d'une rêverie appuyée sur la signifiance de l'écriture.

«En passant dans un bois, elle rencontra compère le loup... ». Rien de la photographie qui accompagne le récit ne correspond mot pour mot à la légende (fig. 11) : ni le chaperon rouge du conte noirci par l'image, ni le loup changé en automobile, ni le bois remplacé par le pavé inégal d'une rue... Et cependant persiste d'un médium à l'autre une cellule narrative commune (un passage et une rencontre) autour de laquelle s'enroulent des formes en écho, des matériaux à rêver pour ce qui les assemble : la proue d'une voiture semblable à la gueule luisante d'une bête, le pavement comparable à de larges écorces...

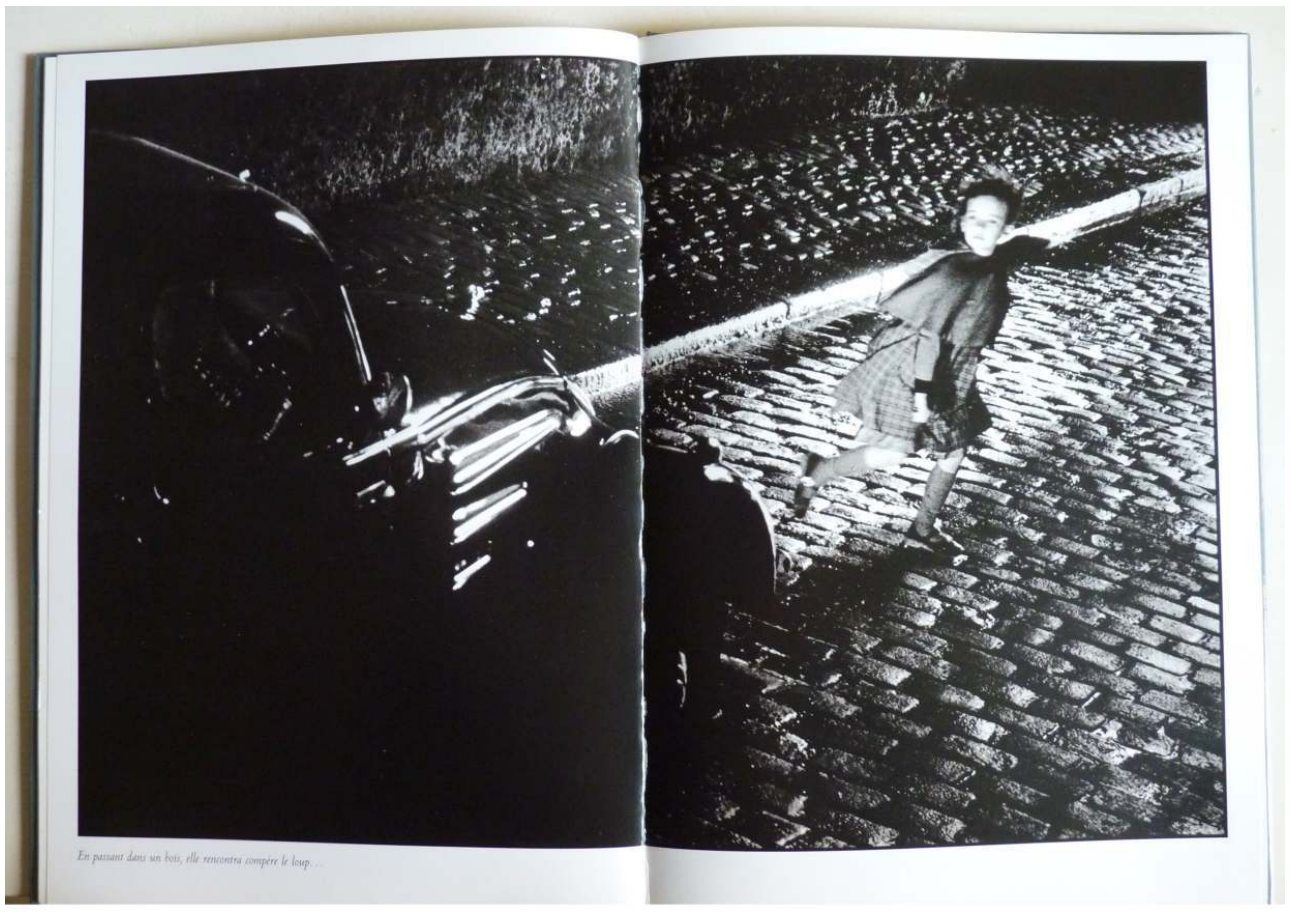

Fig. 11 «En passant dans un bois, elle rencontra compère le loup... », @ Sarah Moon 
Revenons à l'icône matricielle du montage placée au tout début de l'album (fig. 6). Elle établit les deux principes phénoménaux du contact entre texte et image puisqu'on y voit les deux éléments hésiter entre détachement et adhérence. De façon à évoquer plus précisément encore cette double opération de transposition aspectuelle à laquelle travaille Sarah Moon, l'une des premières photographies montre le petit chaperon posant devant une toile peinte (fig. 12). Cette toile est le support des songes et de l'illusion plaqués sur la réalité, elle est aussi le voile de la fiction suspendu en l'air, l'écran de projection d'un drive-in improvisé, une page d'écriture ayant dilué son encre dans le paysage, ou un papier de tirage photographique démesurément agrandi... Les plans de la rue et de la toile parviennent alors à se confondre ou plutôt ils se prolongent grâce aux correspondances visuelles. À quel monde appartient la fillette ? À quel endroit se situe-telle sinon à la lisière des formes, en cette zone fragile et intense où elles se relancent et où leurs différences s'estompent?

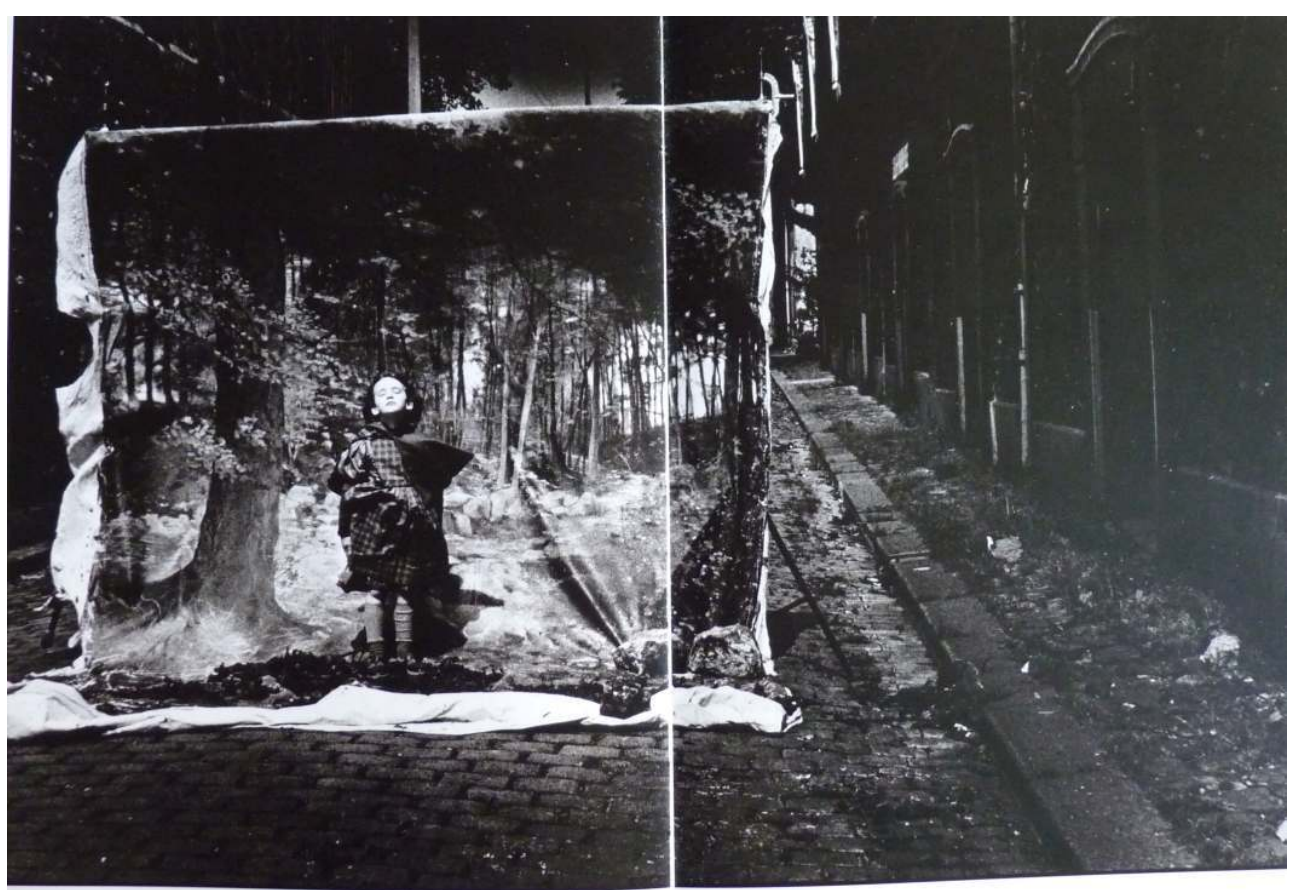

Fig. 12 : Le petit chaperon posant devant une toile peinte, (c) Sarah Moon

La relecture de Sarah Moon assimile donc le rythme de la saccade. Saccade qui définit le battement de son regard passant du texte à l'image et de l'image au texte et s'immobilisant, comme gouverné par un hasard nécessaire, sur ce qui retient le regard dans l'écriture et accroche l'imaginaire. C'est aussi que le récit lui-même oblige à la saccade. Car il est sous le coup d'un danger permanent ; le loup, pulsion sexuelle, est une force disruptive. Il faut alors pour la photographe faire rayonner dans chaque image la splendeur de l'enfance et mobiliser les puissances ténébreuses qui s'acharnent à la déchirer et à jouir d'elle. Les images, cernées par la mort, sont des éclats de beauté et des attaques, selon l'ambiguïté, évoquée par Sarah Moon elle-même, de son propre geste de prise qui soustrait et attrape, enlève et sauve. On repèrerait encore une allusion à la saccade qui correspond à la discontinuité du montage photographique et qui définit son tempo particulier dans les motifs des pavés ou des rails (fig. 13) ; ils induisent la secousse d'un transport (en voiture, tramway ou train) qui emporte certainement vers l'abîme et, en tout cas, vers l'ailleurs. 


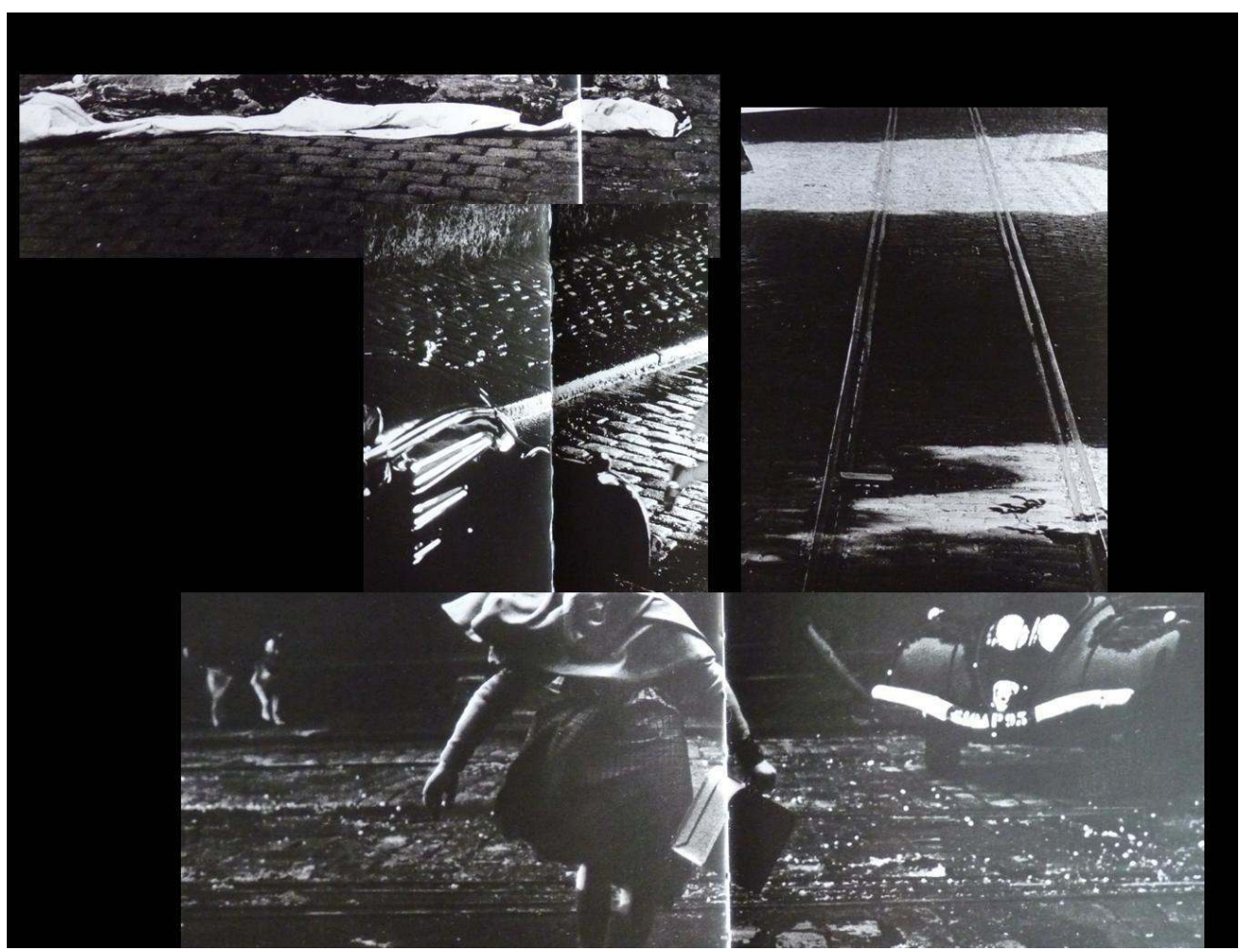

Fig. 13 : Motifs des pavés et des rails, @ Sarah Moon

18 La saccade est enfin le régime de temporalité qui sous-tend l'album. Quand il théorise l'image dialectique, Walter Benjamin ne détermine pas ce qui raccorde le présent et le passé selon l'ordre chronologique; il entend déployer un autre rapport des temps dans lequel le Passé fulgure à travers le Maintenant. À la place de l'illusoire continuité qui souderait les moments, il met en valeur des images qui adviennent par saccades en redonnant l'Autrefois dans le retentissement du Maintenant, ce que Sarah Moon, pour sa part, éprouve comme une «mise à jour » du passé : "Une image [...] est ce en quoi l'Autrefois rencontre le Maintenant dans un éclair pour former une constellation " (Benjamin 478).

19 Le Petit Chaperon rouge résulte de cet enchevêtrement des temporalités qui est aussi migration, dialogue ouvert à la création. On l'a déjà remarqué, l'album obéit à la succession des heures : une horloge en indique la course. Mais aux deux bornes du récit, les aiguilles semblent ne pas avoir bougé (fig. 7), à moins qu'obéissant aux lois de la tragédie, douze voire vingt-quatre heures se soient en réalité écoulées. Le temps a et n'a pas bougé. Dans cette suspension ou cette complexification de la durée, il produit son œuvre : autant celle du conte en images qui avance vers sa fin que celle du dispositif du texte et de la photographie qui sculpte le volume temporel, démultiplie, au gré des allées et venues du chaperon dans les rues et sur les chemins, la circulation entre passé et présent au sein de la mémoire.

20 La petite passante chaperonnée, vagabonde ou affolée, va à travers la ville, elle musarde ou se soustrait à la menace. Elle entraîne avec elle des flux sensibles qui convertissent le texte en images. Les rails encore installent le travelling des temps, et le motif final du drap (fig. 9), aussi terrible soit-il, ne manque pas d'indiquer, par métaphore, les pliages et les déchirures qui soit rabattent passé et présent l'un sur l'autre soit aident à les faire passer l'un vers l'autre. Le miroir (fig. 14) participe de ce paradigme de l'ouvert: sa 
surface brisée annonce l'attaque contre sa pureté que subira la fillette et la déflagration de son identité tout en aménageant localement de menus interstices, des frontières ténues et fragiles par où se diffusent les lignes temporelles : «Je guette ce que je n'ai pas prévu, j'attends de reconnaître ce que j'ai oublié - je défais ce que je construis - j'espère le hasard et je souhaite plus que tout être touchée en même temps que je vise » (Moon 1991).

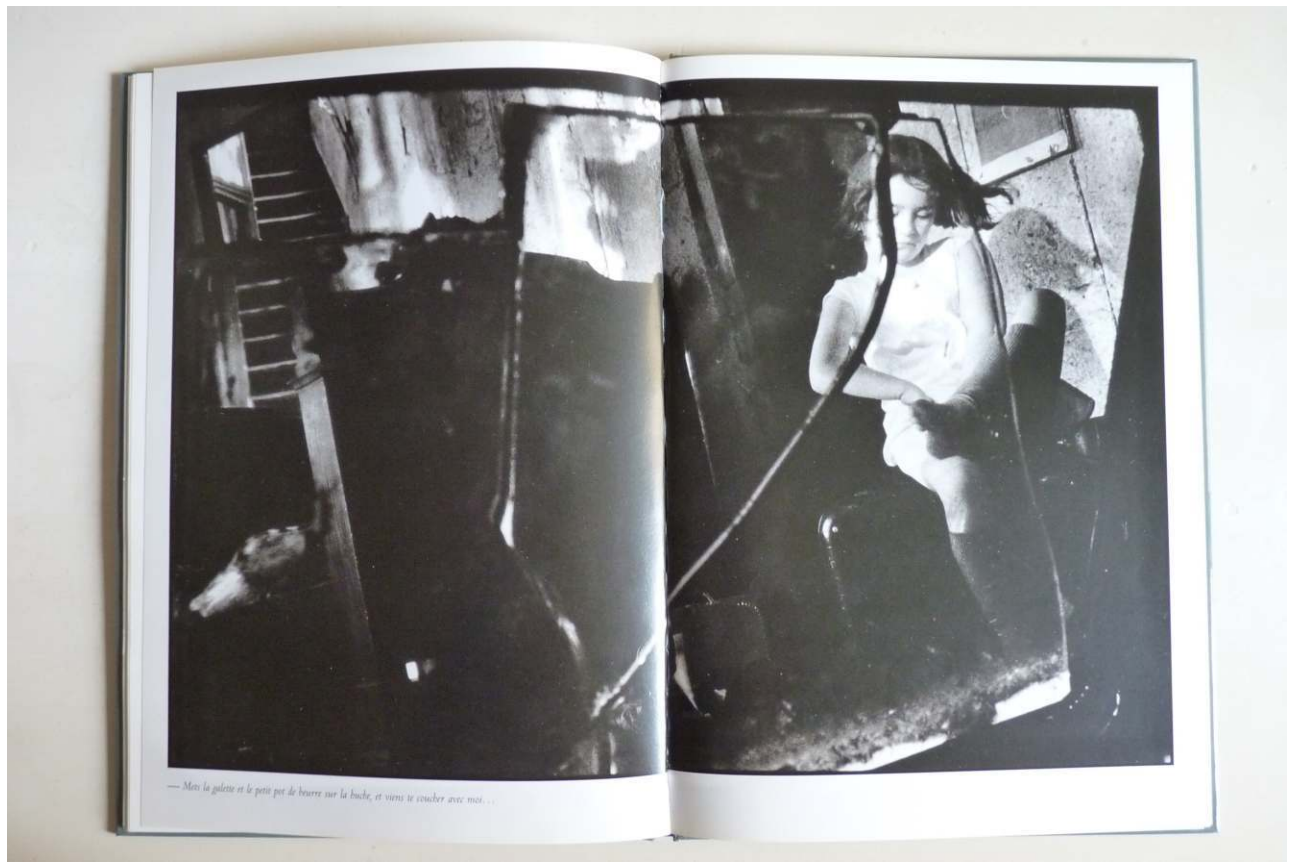

Fig. 14 : Le miroir, (C) Sarah Moon

21 L'« histoire du temps passé »- Perrault nommait ses contes par cette expression - revient traverser les images du temps présent et en recharger la source vitale, par condensation, attraction et expansion. Mais le temps de la photographie lui-même n'est plus que du passé survivant et ce qu'il a enregistré est déjà arrivé, comme il arrivera encore car sa mélancolie est éternelle. Chaque image est un carrefour étoilant en tous sens ses directions, au pli de l'avoir été et de l'être-là.

Quant au référent temporel interne aux images, l'on voit bien qu'il cherche à se situer quelque part dans l'Histoire et ses effroyables remous. La voiture lupine notamment (fig. 15) cristallise un effet de réel et elle est un embrayeur de mémoire. Elle tire l'ambiance du conte, sans la localiser avec exactitude, vers la terreur des temps de haine; le retour insistant des rails serait encore, allusivement, celui des convois de la mort. Mais Sarah Moon ne réinscrit pas sa lecture dans des circonstances ou dans une époque clairement datables, ce qui l'amènerait à la «banalité » de l'anachronisme. Elle traque le temps hors du temps, un destin du texte qui l'engage vers les images. En ce sens seulement l'anachronisme est à ses yeux littéralement vivant et vitalisant. 


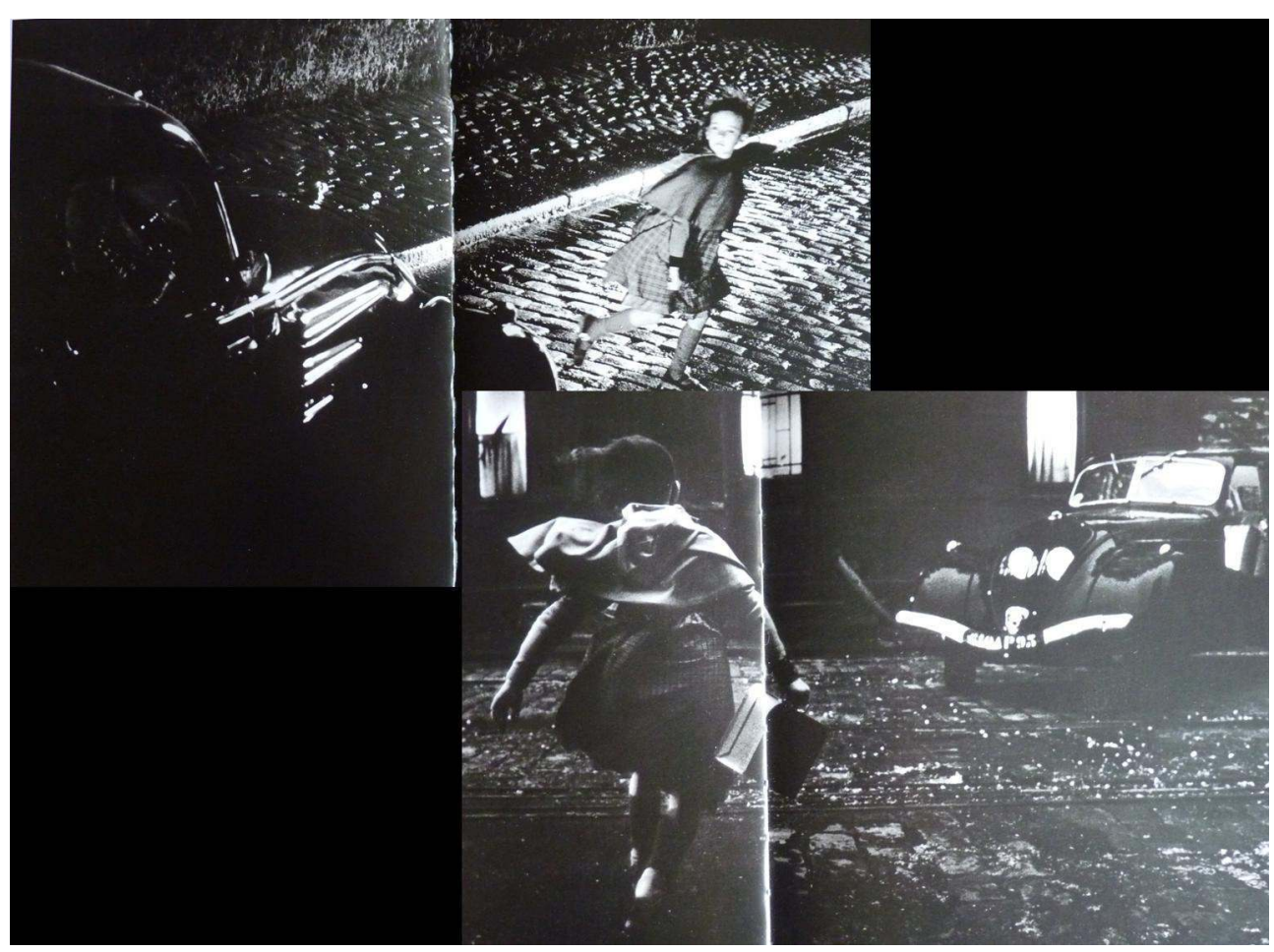

Fig. 15 : La voiture lupine comme embrayeur de mémoire, (c) Sarah Moon

Sarah Moon n'a pas voulu, on l'aura compris, moderniser le conte de Perrault ou le transposer dans quelque reconstitution d'époque. Le montage, mettant à profit les ressources de la scansion et du passage, souligne la manière dont les niveaux de temps se rencontrent, s'annoncent et se déclinent. Les images sont des visions prophétiques du texte, qu'actualise tel ou tel moment de l'histoire où on pourra le reconnaitre ou en reconnaître la portée cauchemardesque, et le texte se retrouve rétrospectivement dans les images, à la fois même et autre. Les photographies ne sont donc pas les souvenirs d'un passé identifiable, qui aujourd'hui nous ramènerait directement et nettement à un hier. Pour reprendre la formule de Walter Benjamin, ce que les images font chatoyer de la moire des histoires, c'est l'aura, quelque chose qui vient de loin, d'un arrière-fond, d'une profondeur de champ, et qui survient au premier plan, comme à jamais dans le présent, afin de nous ravir et nous troubler.

En bloquant la course, tout en l'incitant, l'image tremble, la perception se brouille ; la photographie est alors presque systématiquement gagnée par des zones de flou qui s'ajoutent, pour opacifier la représentation, aux vastes à-plats des ombres, dans le prolongement de toutes les expériences de sfumato qui sont la signature si caractéristique de la lumière et du grain chez Sarah Moon. Le vent accroché à ses cheveux, son vêtement qui se soulève et vogue en l'air (fig. 16), le petit chaperon apparaît et disparaît, ou mieux l'enfant se tient au point médian entre surgissement et dissipation, sur le vif du mouvement qui dépose sur elle sa traîne. 


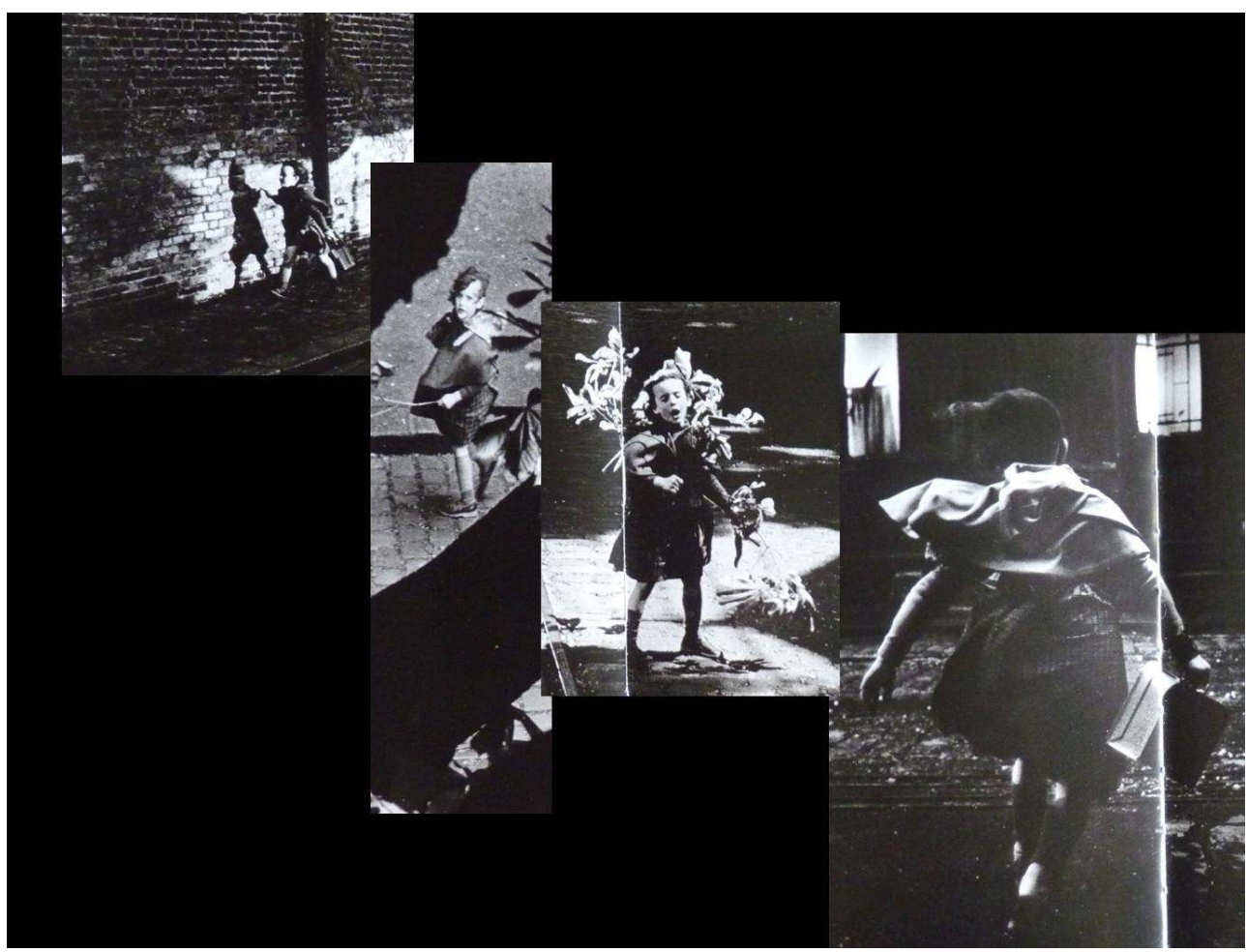

Fig. 16 : L'enfant se tient au point médian entre surgissement et dissipation, sur le vif du mouvement, (c) Sarah Moon

Le flou du visage, du corps, des tissus et finalement par contiguïté de la scène entière recèle en lui l'état sensible et esthétique des images et de ce qui les a engendrées. Il préserve le dynamisme de la fascination à l'instant de son impulsion, mêlé de la conscience de la précarité. La perte et la mort imprègnent l'image qui, en même temps, résiste en commémorant la merveille des gestes de la fillette toujours apparemment tendue vers d'autres déplacements. Il est nécessaire, pour être fidèle à la sensation issue du conte, que l'image soit légère comme l'enfance (frôlant néanmoins l'absence et le dangereux néant), mais il faut aussi qu'elle ait suffisamment de poids pour rappeler la gravité de ce qui est raconté et marquer l'empreinte de ce songe alourdissant l'émotion. La toile peinte devant laquelle Morgan se dresse ou attend (fig. 17), un pan de sa cape soufflé par la bourrasque, le nez retroussé, est ainsi lestée de pierres. Le flou est le signe évasif et prégnant de cette lourdeur aérienne. 


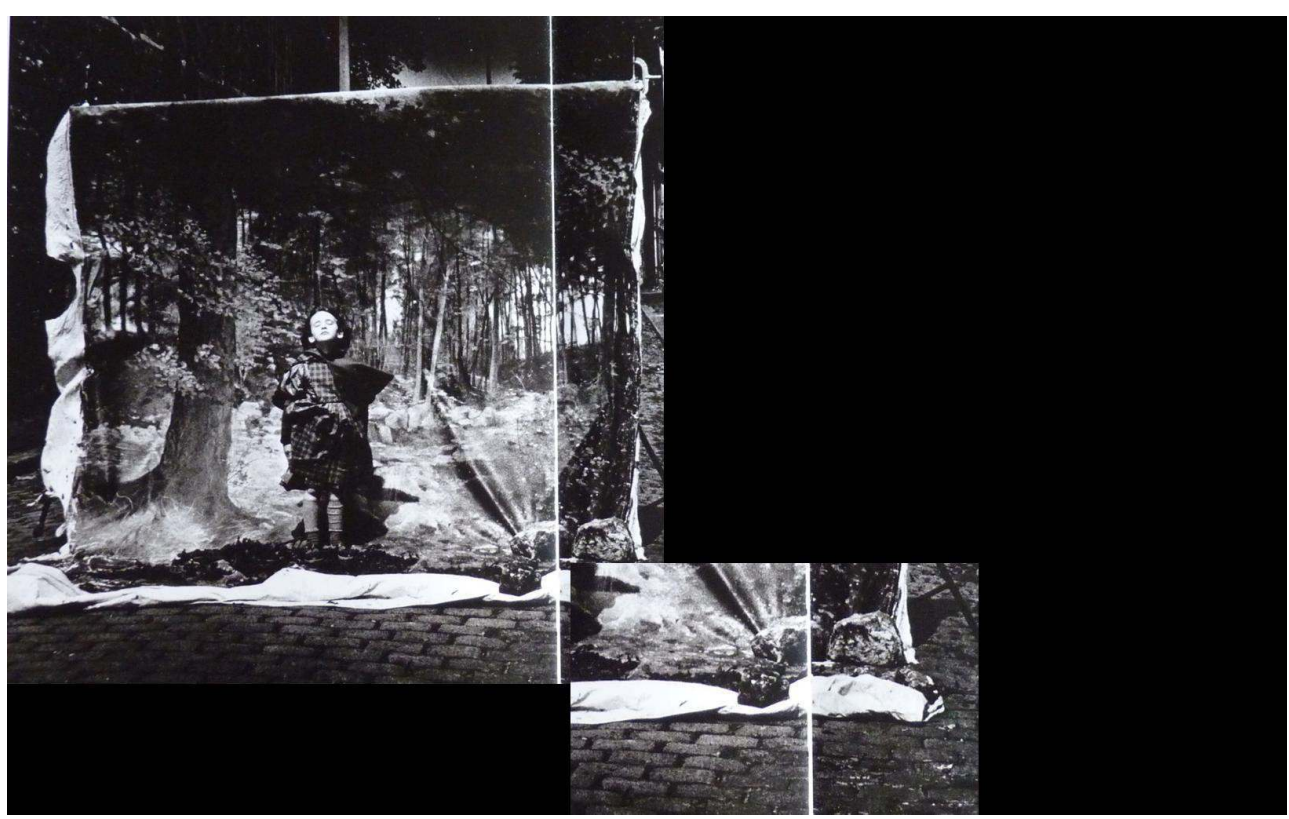

Fig. 17 : La toile peinte devant laquelle Morgan se dresse ou attend est lestée de pierres, @ Sarah Moon

26 La fillette, effigie de l'image, assume d'être une passante et, à l'instar de la photographe, de «brouiller les pistes » (Moon 1991), de flâner comme pour vaporiser la réalité. Sarah Moon pourchasse son mouvement, avec la volonté d'accepter de ne jamais le posséder tout à fait et de témoigner qu'il est irréductible. L'on comprend donc qu'elle ait pu, des années plus tard, repartir du conte pour prendre et relancer d'autres images (un nouvel album, un film), inépuisablement. Le loup, au contraire, aspire à l'arrêt définitif, il entend aller à l'essentiel, mettre un terme sans délais au récit : il prend le plus court chemin et veut créer l'ultime scène, gueule ouverte, au comble de l'ombre. L'image agit tout différemment, même si l'œil avide de la photographe épie son modèle et capture sa lumière. La louve est aussi protectrice, elle enveloppe de son regard l'éphémère et le fragile. L'image capture la jeunesse mais pour étirer, ralentir sa beauté; elle préfère le détour du rêve à la course prédatrice. Voilà encore ce que secrète le flou. Il met l'accent mais cet accent est proche d'une caresse - sur la transformation des qualités et des substances. Il réintroduit une liquidité archaïque de l'image ou bien encore une certaine sensorialité aérienne, en tout cas un essor qui extrait une force génésique des formes et en elles.

27 L'une des premières images surprend le petit chaperon rouge qui touche du doigt un mur (fig. 18). Elle semble y diffuser l'encre de son ombre tout en peignant une coulée de lumière qui avance sur le réel. Et autour d'elle, c'est l'intégralité du paysage urbain qui devient son nimbe : les reflets d'arbres et de toits, le grain du pavement émanent de sa présence fugace. Son vêtement, ses cheveux, bougés par le vent que bloque l'image à l'instant du kairos, se disséminent dans l'espace: ils foisonnent dans les frondaisons des arbres ou ils redoublent l'ondoiement dessiné par le devant d'une voiture (fig. 19). 


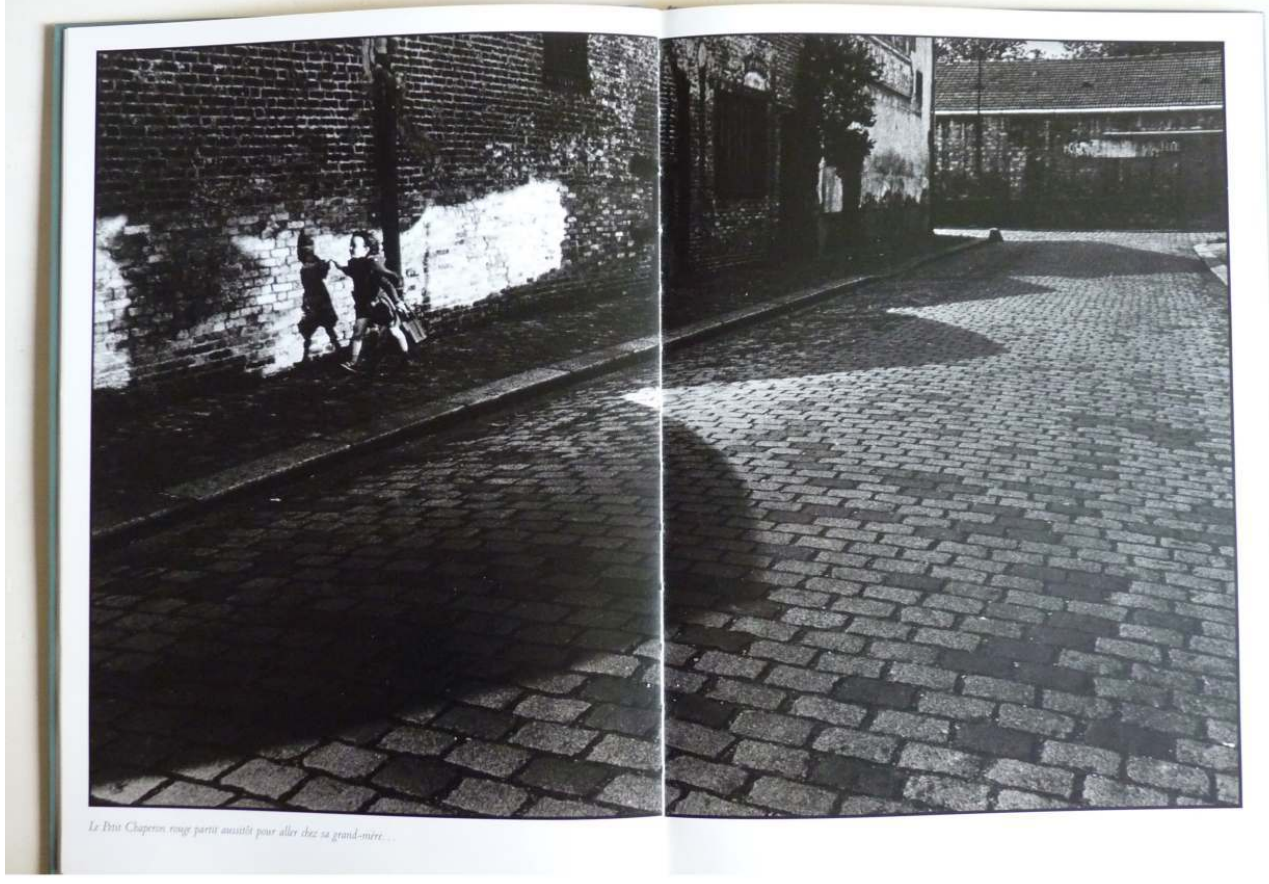

Fig.18 : L'intégralité du paysage urbain devient son nimbe, @ Sarah Moon

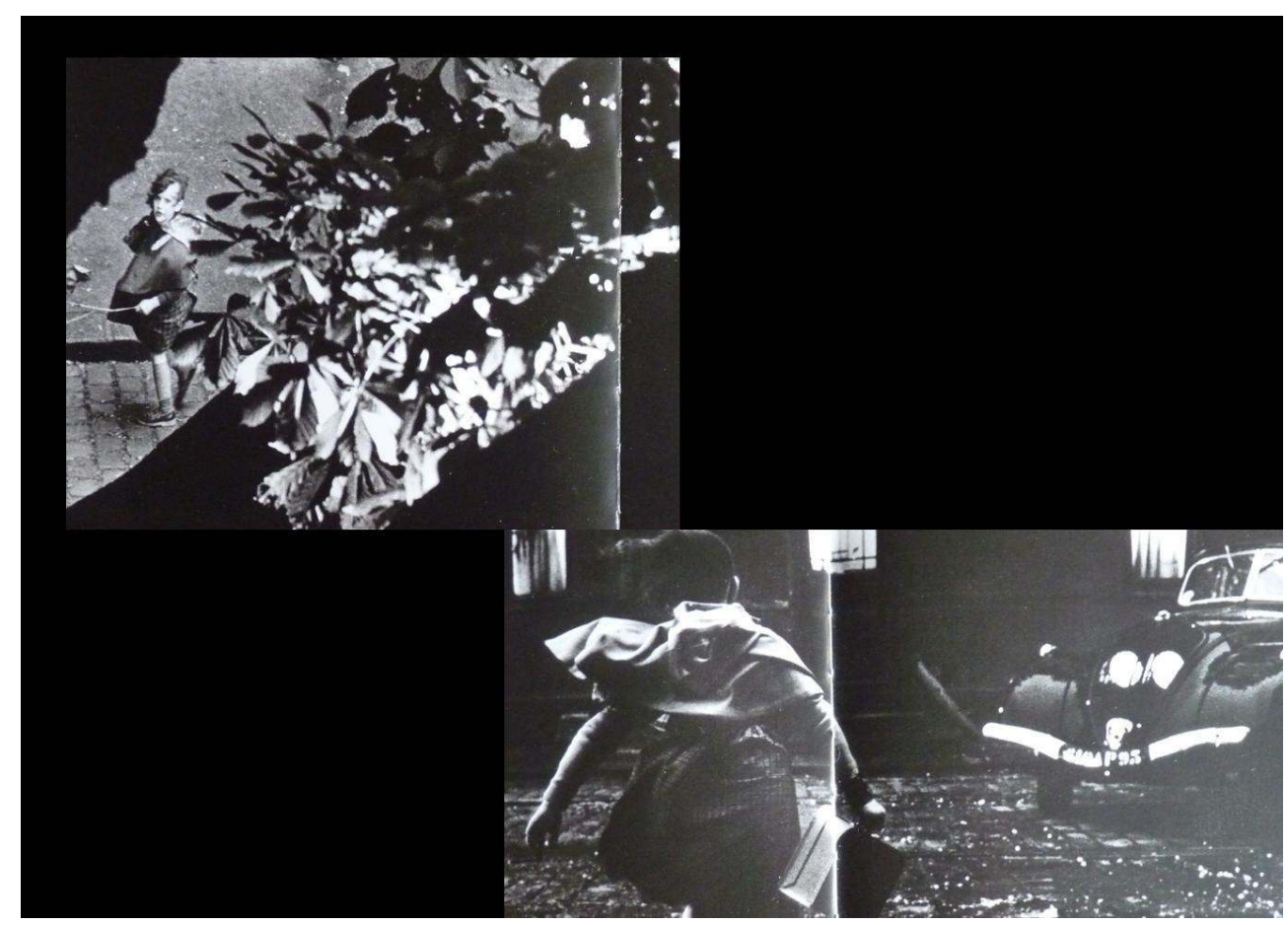

Fig.19: Son vêtement et ses cheveux se disséminent dans l'espace, @ Sarah Moon

Chez Sarah Moon, contrairement à Perrault, le chaperon ne cueille ni papillons ni bouquets de fleurs: la fillette brandit des branches touffues et elle se noie dans des grappes de feuilles qui en sont les substituts (fig. 10). Car elle est elle-même papillon et feuille, emportée par l'air et battant à sa mesure. Le chaperon rouge est une gerbe de métaphores. Les associations imaginaires dont elle est le corps conducteur prennent leur revanche sur ce qui s'échine à détruire l'enfance, manière d'imposer à la mort le pouvoir 
des résurrections. Pour Sarah Moon, les saccades de la photographie sont des battements d'ailes : «je ne peux m'empêcher de penser à ce qu'écrivait Dubuffet : «Il se peut qu'en donnant à l'œuvre existence, on en perde le meilleur. Elle devient alors le papillon épinglé qui a cessé de voler, qui a cessé somme toute d'être papillon » (Moon 2008, 379, tome 3).

Les images pourraient ne vouloir retenir, obsessionnellement, que le désastre de l'existence meurtrie, que la sidération de l'agression et la souillure de l'enfance amèrement sacrifiée. Mais toutes ensemble, par le travail dialectique du montage, elles parviennent à récupérer l'énergie du sensible qu'elles se transmettent et font pleinement vivre, même si l'image ressemble à une fuite: "Souvent j'envie ceux qui savent photographier la vie. Moi, je la fuis [...] » (Moon 1991). La photographie de Sarah Moon explore, par à-coups, par spasmes, la figurabilité de l'image, au sujet de laquelle Eisenstein a pu parler d'aimantation et d'explosion graphiques. Cette alchimie de la figurabilité, que Sarah Moon éprouve comme "une quête sans fin" une "course au trésor » (Moon 1991) ou à la chimère (Moon 2008, 87, tome 1), est d'autant plus pathétique et elle est d'autant plus intense que sourdent en elle la disparition et la mort. Le flou rappelle alors, pour suivre Dominique Eddé, que la vitre derrière laquelle Sarah Moon photographie les yeux du monde « est couverte de larmes " (Eddé dans Moon 2008, 234, tome 2) ; elle est embuée. Cependant la violence imposée par l'ombre à la lumière fait advenir, en chaque plan d'image trempée dans le révélateur du texte, des formes et des substances mutables, spectrales, évasives, buissonnières. La bonne heure pour cette évasion aux pays des fantômes est l'heure du loup, intermédiaire, tremblante et séminale, vers cinq heures du soir. À ce moment s'éveille un régime poétique de turbulences visuelles et de transmutations des matériaux (traîne du vent, fouillis des feuilles, grain du pavement, noise des tissus...) qui complètent l'effroi par l'effort de la beauté.

Sur le visage flou du petit chaperon (fig. 20), la lumière nappée de l'image émue vient se mêler aux clairs-obscurs aiguisés par la pulsion que le loup exacerbe. Mais elle parvient à écarter l'ombre en ses franges et elle irradie. Quant aux plis du drap rimant avec les lignes dentelées, mordantes du miroir qu'a précédemment brisé l'avidité du désir (fig. 21), ses arrêtes se combinent à l'offrande de qualités plus douces et plus tendres, susceptibles d'autres expansions sensorielles et qui se sont déjà manifestées aux images précédentes (fig. 22) : dans le drapé de la cape, le scintillement des rails, l'ovale légèrement effrangé des feuilles, et même le halo de l'éclairage projeté par les phares de la voiture, pourtant hostile. Le luminisme de Sarah Moon règle en tension et selon de permanents échanges l'ombre et la lumière: aimantation, intrication, brouillage, attaques réciproques, brillances et opacités, points d'accumulation ou lignes de dissipation..., la gamme est infinie. En sorte qu'il est bien difficile de savoir si l'émotion l'emportera sur la prédation ou si, plus étrangement encore, du point de vue des formes et des sensations en tout cas, tendresse et pulsion ne résultent pas d'une séduction réciproque. C'est ainsi que chez Sarah Moon, l'image prend et tient, en cet équilibre frémissant. 


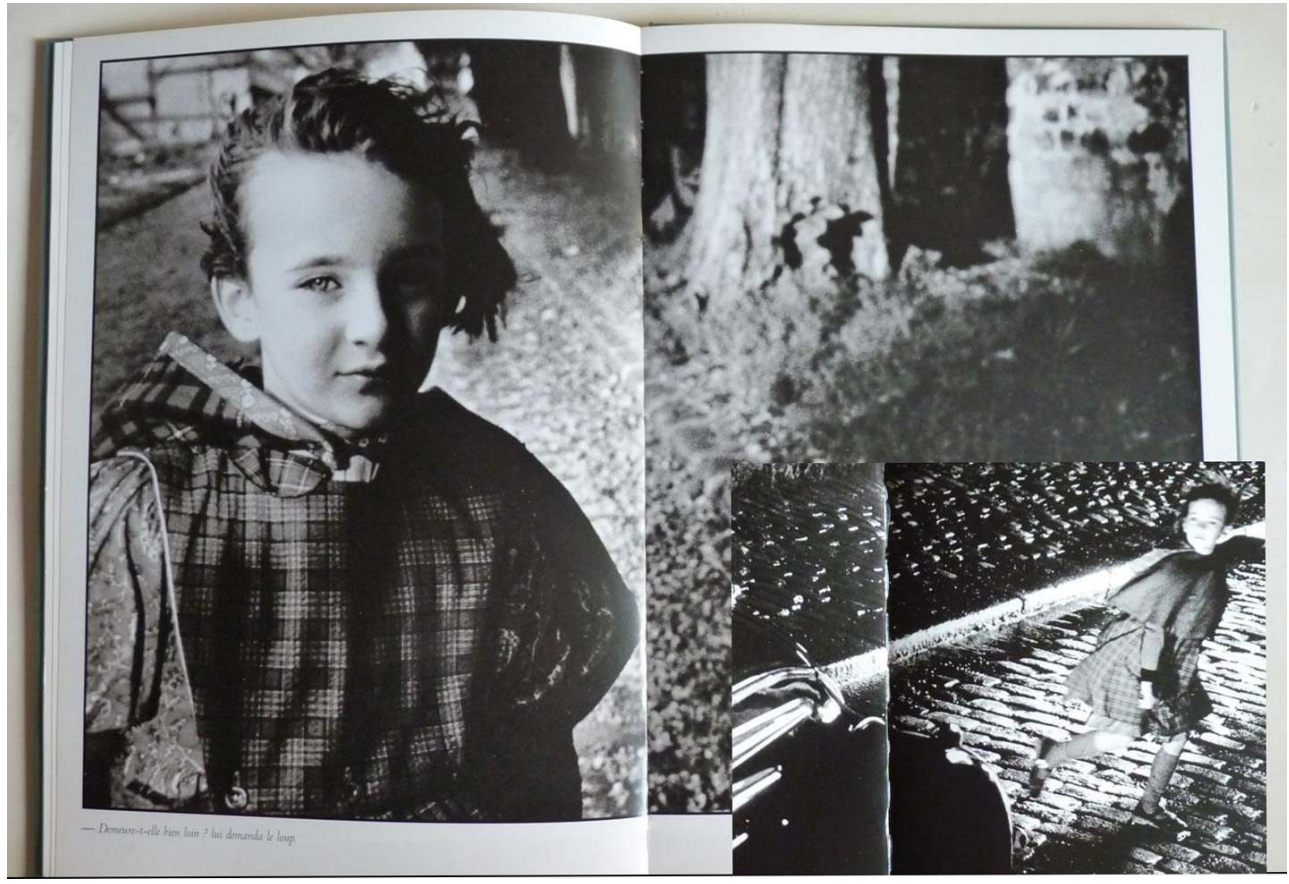

Fig.20 : Clairs-obscurs sur le visage du petit chaperon, ( ) Sarah Moon

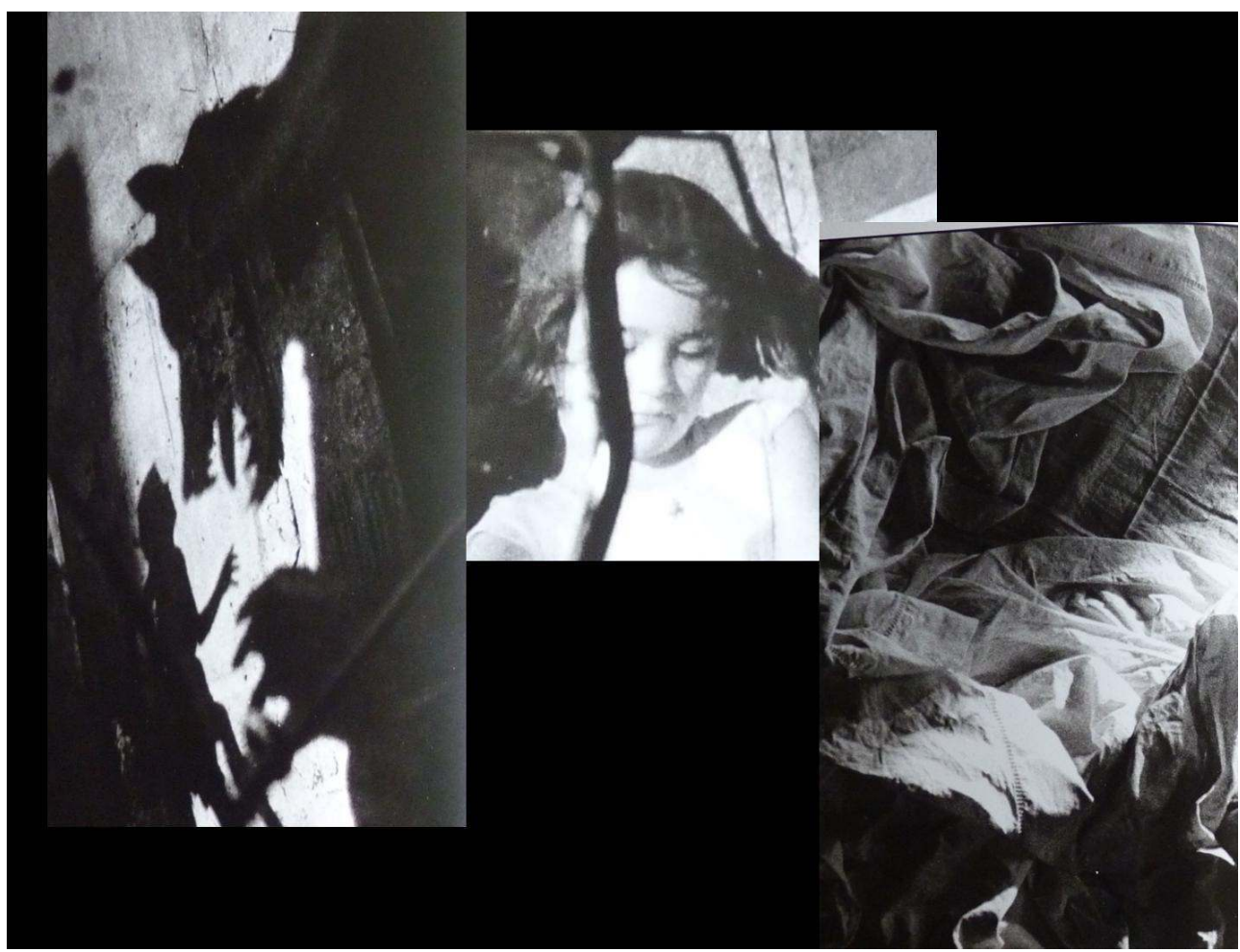

Fig.21 : Lignes dentelées et mordantes, @ S Sarah Moon 


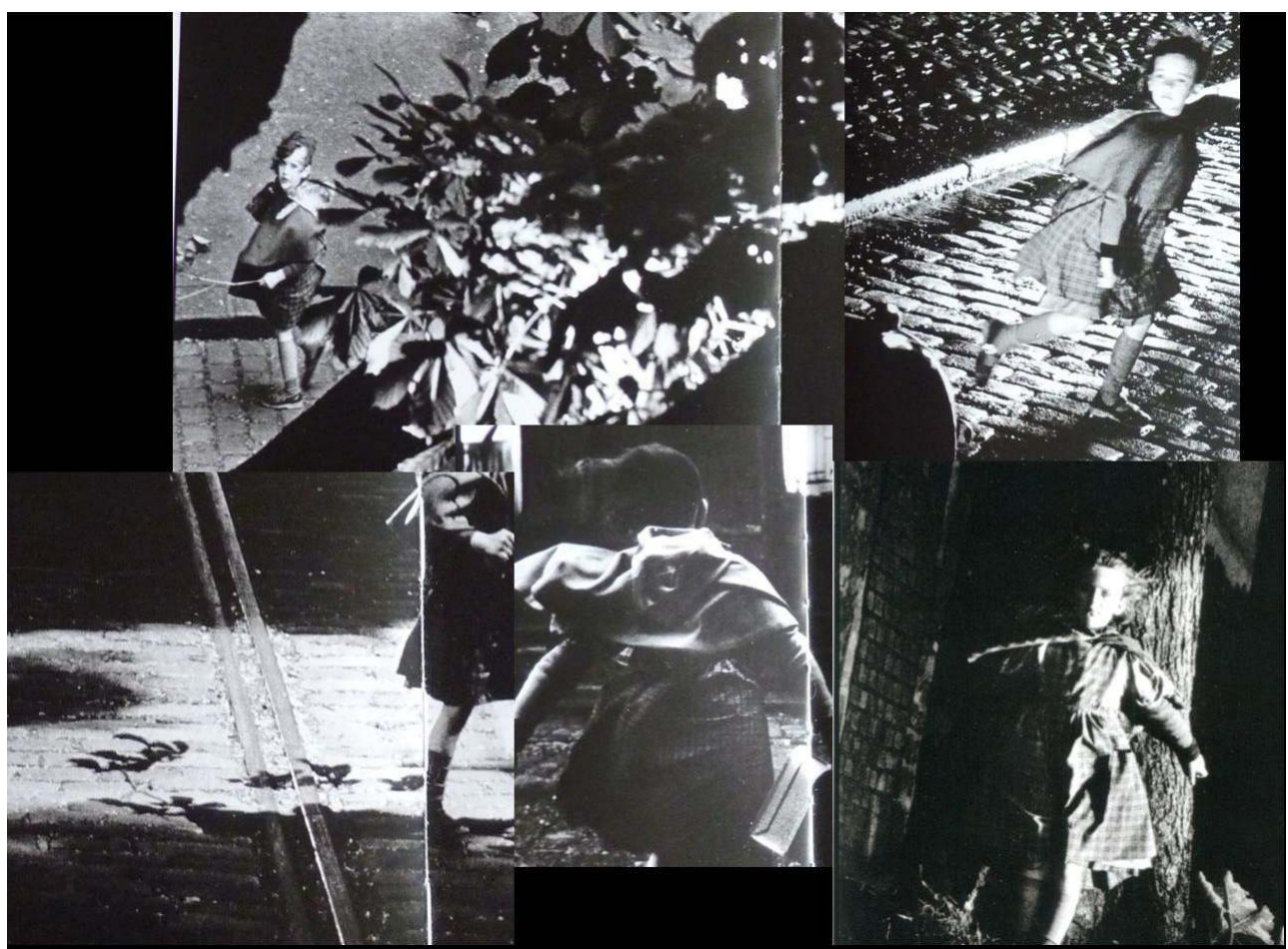

Fig.22 : Drapé de la cape, scintillement des rails, ovale légèrement effrangé des feuilles, halo de l'éclairage projeté par les phares de la voiture, @ Sarah Moon

«Il était une fois »: par ces mots, démarrent d'un même pas rétrograde récit et images. Dans ce «il était une fois », l'universel du conte agrandi en mythe se resserre, on le sent bien, malgré la pudeur des images, sur l'expérience la plus intime, la plus autobiographique qui ne vise pas cependant à retenir l'image dans un territoire exclusivement privé.

«Il était une fois » fonde un récit, écrit par un autre, Perrault, et même raconté bien avant lui, mais qui tressaille également d'une histoire qui appartient en propre. Cette histoire glisse de la communauté des lecteurs à laquelle veut parler le conte vers la singularité d'une certaine solitude dans l'écoute des fictions. Le récit personnel de Sarah Moon, qui retentit dans le conte, échappe pourtant ; il est indicible finalement, incomplet, non reconstituable malgré les images qui lui courent après. Nous n'en savons que les traces ténues et ces traces tout regard peut en être ému. Le récit en images ne cesse alors, à travers les yeux clairs de la petite Morgan déguisée en chaperon, de questionner le regard insaisissable et un peu myope de celle qui prend ses photos et qui, à travers son objectif, cherche le temps, et elle-même, avec le désir, plus fort que la solitude mélancolique, de le transmettre.

Le flou est la marque de cette mémoire vacillante, intermittente et de l'histoire qu'elle fait trembler. En lui s'adresse le désir d'une mise au point qui, dans le même temps, maintient le virtuel voire l'indécidable ou l'éphémère des rencontres avec soi et le monde, mais qui essaie aussi de retenir ce qu'il y aurait à sauver. Sarah Moon parle de ses photographies comme de rescapées de la nuit; le regard tourné vers ce qui, en fait, a commencé bien avant (au temps vague du «il était une fois»), ses images paraissent à chaque fois les toutes dernières. Au bord de s'exténuer, mangées par cette vapeur qui peut se dramatiser en ombre, elles demeurent en cet état transitionnel qui assure le passage vers d'autres regards, comme un appel à partager l'espoir du salut. 


\section{BIBLIOGRAPHIE}

Agamben, Giorgio. Image et Mémoire. Écrits sur l'image, la danse et le cinéma. Paris : Desclée de Brouwer, 2004.

Barthes, Roland. La Chambre claire. Note sur la photographie. Paris : Cahiers du Cinéma/Gallimard/ Éditions du Seuil, 1980.

Barthes, Roland. «Écrire la lecture ». Euvres complètes. É. Marty (éd.). Paris : Seuil, 1993-1995.

Bellour, Raymond. Le Corps du cinéma. Hypnoses, émotions, animalités. Paris : P.O.L. (coll. Trafic), 2009.

Benjamin, Walter. Paris Capitale du XIX ${ }^{e}$ siècle. Le Livre des passages [1927-40]. Trad. J. Lacoste. Paris : Éditions du Cerf, 1993.

Cérès, Patrick. L'Émotion du cinéma : contribution à l'analyse d'un phénomène. Thèse de doctorat, soutenue en 2003, sous la direction de J.-P. Berthomé, Université de Rennes 2 Haute-Bretagne, UFR Arts, Lettres, Communication.

Moon, Sarah. Souvenirs improbables. Paris : Delpire, 1981.

Moon, Sarah. Vrais Semblants. Paris : Delpire, 1991.

Moon, Sarah. Contacts. Arte France : CNP, KS Visions, 1993.

Moon, Sarah. 1, 2, 3, 4, 5. Paris : Delpire, 2008.

Perrault, Charles / Moon, Sarah. Le Petit Chaperon rouge. Paris : Grasset (coll. Monsieur Chat), 1983. Vally, Hélène. « Penser et analyser le flou cinématographique : le parti-pris du sensible ». Textimage. Varia 3, 2003, URL : https://www.revue-textimage.com/07_varia_3/vally1.html (dernière consultation le 27 novembre 2017)

\section{NOTES}

1. «Par la marque de quelque chose, la photo n'est plus quelconque. Ce quelque chose a fait tilt, il a provoqué en moi un petit ébranlement, un satori, le passage d'un vide (peu importe que le référent en soit dérisoire) »(Barthes 1980, 80-81).

2. Le Petit Chaperon rouge est la première étape d'une série d'adaptations de contes: Circus en 2003 (tiré de La Petite Marchande aux allumettes), L'Effraie en 2004 (tiré de Le Petit Soldat de Plomb), Le Fil rouge en 2005 (tiré de La Barbe Bleue), La Sirène d'Auderville en 2007 (tiré de La Petite Sirène). 


\section{RÉSUMÉS}

Au début des années 80 , Sarah Moon choisit, au moyen de la photographie, de « re-conter» Le Petit Chaperon rouge de Charles Perrault. Elle en reproduit le court récit, légèrement remanié, et intercale en lui une série de clichés en noir et blanc. À travers ces images ajoutées, Sarah Moon lève dans le conte les ressources d'un autre temps, qu'il recèle déjà, et dont elle libère cliché après cliché la potentialité. Ces photographies frappantes et, à la fois, évanescentes d'une jeune passante pourchassée sont celles d'un passage des temporalités où se rencontrent, sur fond d'effroi, histoire intime et Histoire universelle. Nœuds de temps en leur point tragique, les images pourraient ne vouloir signifier, obsessionnellement, que la violence de l'existence meurtrie et déchirée, que la sidération de l'agression et la souillure de l'enfance amèrement perdue. Mais toutes ensemble, par le travail du montage, elles parviennent aussi à faire sourdre une énergie du sensible qu'elles se transmettent et font pleinement vivre.

At the beginning of the 1980s, Sarah Moon chooses, by means of photography, to "re-tell" Charles Perrault's Little Red Riding Hood. Re-printing a slightly altered version of this short tale, she inserts in it a series of photographs in black and white. Through these added images, Sarah Moon adumbrates the presence of another layer of time, already contained yet hidden in the tale, and releases its potentialities, photograph by photograph. These photographs, both striking and evanescent, all represent a young girl taking flight before some unknown danger: in those scenes, different temporalities come together, as intimate history and universal History collide against a background of fear and tension. It might be that those images - nodes of time, in their tragic concentration - only mean to show an obsession with the violence of ravaged and torn existence, or a form of bafflement at the idea of childhood assaulted, corrupted, and sadly lost. But all together, through the operation of montage in the book, they also succeed in exuding, transmitting, and actualising a particular sensorial energy.

\section{INDEX}

Mots-clés : photographie, conte, montage, figurabilité

Keywords : photography, tale, montage, figurability

\section{AUTEURS}

\section{OLIVIER LEPLATRE}

Olivier Leplatre est professeur de littérature du XVII siècle à l'Université Jean-Moulin Lyon 3. Il travaille en particulier sur les rapports entre les textes et les images sous l'Ancien Régime et au $\mathrm{XX}^{\mathrm{e}}$ siècle, et est co-fondateur de la revue en ligne Textimage. 